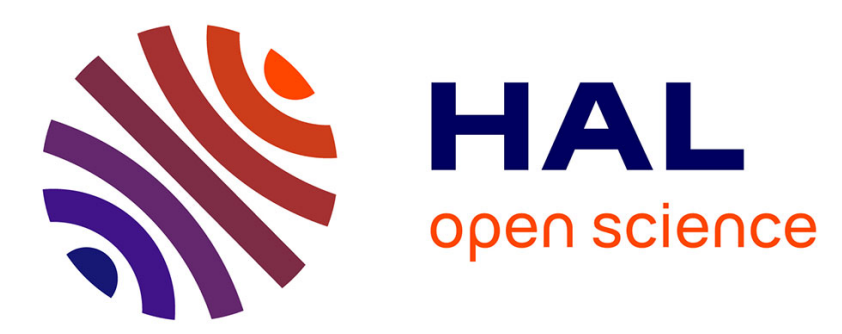

\title{
Optical Shielding of Destructive Chemical Reactions between Ultracold Ground-State NaRb Molecules
}

\author{
T. Xie, Maxence Lepers, R. Vexiau, A. Orbán, O. Dulieu, N. Bouloufa-Maafa
}

\section{To cite this version:}

T. Xie, Maxence Lepers, R. Vexiau, A. Orbán, O. Dulieu, et al.. Optical Shielding of Destructive Chemical Reactions between Ultracold Ground-State NaRb Molecules. Physical Review Letters, 2020, 125 (15), 10.1103/physrevlett.125.153202 . hal-03047050

\section{HAL Id: hal-03047050 https://hal.science/hal-03047050}

Submitted on 8 Dec 2020

HAL is a multi-disciplinary open access archive for the deposit and dissemination of scientific research documents, whether they are published or not. The documents may come from teaching and research institutions in France or abroad, or from public or private research centers.
L'archive ouverte pluridisciplinaire HAL, est destinée au dépôt et à la diffusion de documents scientifiques de niveau recherche, publiés ou non, émanant des établissements d'enseignement et de recherche français ou étrangers, des laboratoires publics ou privés. 


\title{
Optical Shielding of Destructive Chemical Reactions between Ultracold Ground-State NaRb Molecules
}

\author{
T. Xie $\odot,{ }_{1}^{1}$ M. Lepers $\odot,{ }^{2}$ R. Vexiau, ${ }^{1}$ A. Orbán $\odot,{ }^{3}$ O. Dulieu $\odot,{ }^{1}$ and N. Bouloufa-Maafa ${ }^{1}$ \\ ${ }^{1}$ Université Paris-Saclay, CNRS, Laboratoire Aimé Cotton, 91405 Orsay, France \\ ${ }^{2}$ Laboratoire Interdisciplinaire Carnot de Bourgogne, CNRS, Université de Bourgogne Franche-Comté, 21078 Dijon, France \\ ${ }^{3}$ Institute for Nuclear Research (ATOMKI), H-4001 Debrecen, Pf. 51, Hungary
}

(Received 3 June 2020; accepted 9 September 2020; published 8 October 2020)

\begin{abstract}
We propose a method to suppress the chemical reactions between ultracold bosonic ground-state ${ }^{23} \mathrm{Na}^{87} \mathrm{Rb}$ molecules based on optical shielding. By applying a laser with a frequency blue-detuned from the transition between the lowest rovibrational level of the electronic ground state $X^{1} \Sigma^{+}\left(v_{X}=0, j_{X}=0\right)$, and the long-lived excited level $b^{3} \Pi_{0}\left(v_{b}=0, j_{b}=1\right)$, the long-range dipole-dipole interaction between the colliding molecules can be engineered, leading to a dramatic suppression of reactive and photoinduced inelastic collisions, for both linear and circular laser polarizations. We demonstrate that the spontaneous emission from $b^{3} \Pi_{0}\left(v_{b}=0, j_{b}=1\right)$ does not deteriorate the shielding process. This opens the possibility for a strong increase of the lifetime of cold molecule traps and for an efficient evaporative cooling. We also anticipate that the proposed mechanism is valid for alkali-metal diatomics with sufficiently large dipoledipole interactions.
\end{abstract}

DOI: 10.1103/PhysRevLett.125.153202

Ultracold quantum gases composed of particles interacting at large distances in an anisotropic manner represent promising platforms for studying many-body physics and strongly correlated systems [1-3] for high-impact applications like quantum simulation [4] and quantum computation [5]. Among the possible candidates feature ultracold polar molecules, as their large permanent electric dipole moment induced by an electric field can generate intense long-range anisotropic dipole-dipole interactions (DDIs) [6-12]. However, such studies with ultracold molecules in their absolute ground state are hindered when ultracold chemical reactions occur at short distances, like with the ${ }^{40} \mathrm{~K}^{87} \mathrm{Rb}$ species [13,14]. Even for the nonreactive bosonic ${ }^{87} \mathrm{RbCs}$ [15-17] and ${ }^{23} \mathrm{Na}^{87} \mathrm{Rb}$ [18], or fermionic ${ }^{23} \mathrm{Na}^{40} \mathrm{~K}$ $[19,20]$ species, which were thought to be immune to inelastic losses [21], limited lifetimes of the ultracold molecular samples were recorded. The origin of this loss mechanism is tedious to identify, as the final products are not easily detected [18,22]. The first success in this direction has been recently obtained with the reactive species ${ }^{40} \mathrm{~K}^{87} \mathrm{Rb}[23,24]$.

In Ref. [25] it was suggested that the observed losses in $\mathrm{NaK}+\mathrm{NaK}$ ultracold collisions come from the electronic excitation caused by trapping lasers. This hypothesis has been confirmed in Ref. [26] where the dominant loss for ${ }^{87} \mathrm{RbCs}$ arises from the fast optical excitation of long-lived bimolecular complexes, while the losses may be significantly suppressed by square-wave modulation of the trap intensity. However, this is still not enough to prepare longlived ultracold molecular samples in the absolute ground state.
Instead, one can use an electromagnetic (em) field without any additional field to control the long-range interactions between two ground-state bialkali molecules $\mathrm{AB}$ in their lowest vibrational level $v=0$. A microwave

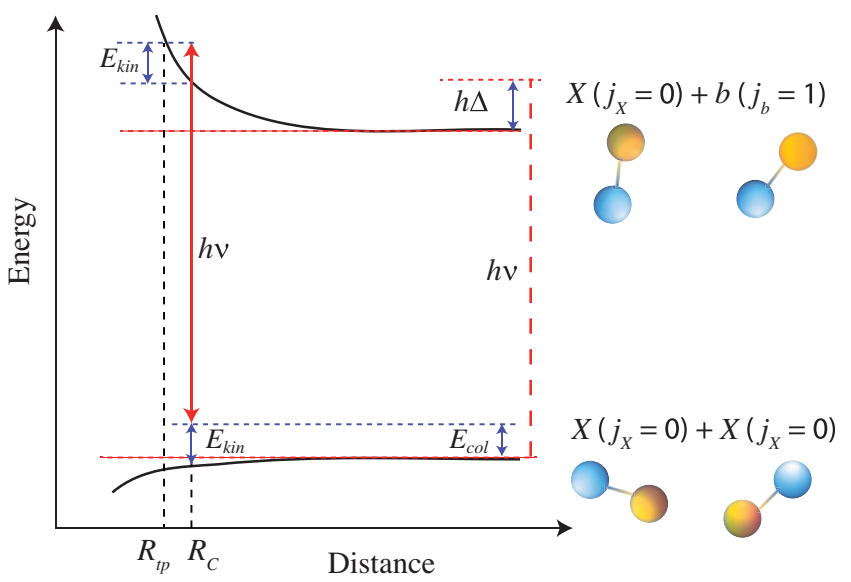

FIG. 1. Classical picture of the optical shielding. Two groundstate molecules collide with energy $E_{\text {coll }}$. At the Condon point $R_{C}$ (conserving the local kinetic energy $E_{\text {kin }}$ ) the pair absorbs a photon of energy $E=h \nu$ to reach a repulsive long-range potential-energy curve of a $\left[X\left(j_{X}=0\right)\right]$ molecule interacting with an excited $\left[b\left(j_{b}=1\right)\right]$ molecule. $\nu=E / h$ is blue-detuned by $\Delta>0$ from the $X\left(j_{X}=0\right)-b\left(j_{b}=1\right)$ transition frequency $\nu_{X b}$, i.e., $E=h \nu=h \nu_{X b}+h \Delta$. $R_{\mathrm{TP}}$ is the classical turning point for the relative motion in the upper curve. The molecules are thus prevented from reaching the reactive zone. Stimulated emission in the excited complex also occurs if the field is strong enough (thus, the double-arrowed red line). 
(MW) field is tuned on the transition between the two rotational sublevels $j=0$ and $j=1$. This engineers repulsive long-range interactions between $\mathrm{AB}$ molecules due to the coupling between the entrance scattering channel $\mathrm{AB}(j=0)+\mathrm{AB}(j=0)$ and the neighboring one $\mathrm{AB}(j=$ $0)+\mathrm{AB}(j=1)[27-30]$. It was found that a significant shielding can only be achieved with a circularly polarized MW field, tedious to implement experimentally [31].

An optical em field couples electronic states of the colliding particles. The proof of principle of such an optical shielding (OS) has been demonstrated for inelastic collisions between ultracold alkali-metal atoms by coupling the ${ }^{2} S$ ground state to the lowest ${ }^{2} P$ excited state [32-38]. The circularly polarized $\left(\sigma^{ \pm}\right)$light was shown to work better than the linear-polarized $(\pi)$ one, but the shielding efficiency was hindered by spontaneous emission from the ${ }^{2} P$ level $[31,36,39,40]$.

Here, we demonstrate the OS efficiency to suppress reactive collisions between ultracold ${ }^{23} \mathrm{Na}^{87} \mathrm{Rb}$ molecules in the lowest rovibrational level $\left(v_{X}=0, j_{X}=0\right)$ of the $X^{1} \Sigma^{+}$ ground state (noted $j_{X}=0$ ) in free space. The principle (Fig. 1) involves a laser with frequency blue-detuned from the transition between the $j_{X}=0$ level and the $\left(v_{b}=0\right.$, $\left.j_{b}=1\right)$ rovibrational level of the excited $b^{3} \Pi_{0}$ state (noted $j_{b}=1$ ), with energy $E / h c=11306.5 \mathrm{~cm}^{-1}$ (or $884.447 \mathrm{~nm}$ ) [41]. This spin-forbidden transition facilitated by the spin-orbit coupling between the $b^{3} \Pi_{0}$ and the $A^{1} \Sigma_{0}^{+}$ excited states ensures that the spontaneous emission does not hinder the OS efficiency. We calculated the bimolecular long-range potential-energy curves (PECs) from our previous developments [42-45] safely neglecting the hyperfine structure of the rotational levels (see Supplemental Material [46]). We found that a PEC correlated to the $\left(j_{X}=0\right)+\left(j_{b}=1\right)$ asymptote possesses a high potential barrier caused by DDI. In contrast with the MW case, OS is efficient for both $\sigma^{ \pm}$and $\pi$ light. Its efficiency monotonically increases with the strength of the $X-b$ optical coupling and is almost temperature independent. OS is not perturbed if the molecules are held in a conventional optical dipole trap with a wavelength far off any resonance reachable from the $j_{X}=0$ level, like $1064 \mathrm{~nm}$ [46].

We solve the time-independent Schrödinger equation describing the two-body collision between ground-state ${ }^{23} \mathrm{Na}^{87} \mathrm{Rb}$ molecules in the presence of the OS field, and no other field. The Hamiltonian for the complex with reduced mass $\mu$ is written as

$$
\begin{aligned}
\hat{H}= & -\frac{\hbar^{2}}{2 \mu R^{2}} \frac{\partial}{\partial R}\left(R^{2} \frac{\partial}{\partial R}\right)+\frac{\mathbf{L}^{2}}{2 \mu R^{2}}+\hat{H}_{\text {rot }} \\
& +\hat{V}(R)+\hat{H}_{f}+\hat{H}_{I},
\end{aligned}
$$

with $\mathbf{L}$ the angular momentum of the mutual rotation of the molecules with the quantum number $\ell$ (the partial wave), and $\hat{H}_{\text {rot }}$ the sum of the rotational Hamiltonian for each molecule. The operator $\hat{V}(R)$ holds for the long-range interaction between the molecules, including the DDI $\left(\propto R^{-3}\right)$ and the van der Waals interaction $\left(\propto R^{-6}\right)$ [42-46]. The laser field Hamiltonian is $\hat{H}_{f}$, and $\hat{H}_{I}$ is the interaction between the complex and the laser field, depending on the Rabi frequency $\Omega$ and the detuning $\Delta$ with respect to the $\left(j_{X}=0\right)-\left(j_{b}=1\right)$ transition energy.

We introduce the symmetrized fully coupled basis functions in the space-fixed (SF) frame $\mid e_{1}, j_{1}, p_{1}, e_{2}$, $\left.j_{2}, p_{2}, j_{12}, \ell, J, M\right\rangle$ to characterize the scattering channels [45]: For $i=1,2$, the quantum numbers $e_{i}=X$, or $b, j_{i}$, and $p_{i}= \pm 1$, respectively, describe the electronic state, the total angular momentum $\mathbf{j}_{\mathbf{i}}$, and the parity of each molecule. If $e_{i}=X$, then $p_{i}=(-1)^{j_{i}}$, while if $e_{i}=b$, both parities $p_{i}= \pm 1$ exist for a given $j_{i}$. The angular momenta $\mathbf{j}_{\mathbf{1}}$ and $\mathbf{j}_{\mathbf{2}}$ are coupled to give $\mathbf{j}_{\mathbf{1 2}}$, itself coupled with $\mathbf{L}$ to give the total angular momentum of the complex $\mathbf{J}$ and its projection $J_{z}$ on the $\mathrm{SF} z$ axis (with quantum number $M$ ).

We use the dressed-state model [55] to account for photon absorption and stimulated emission, introducing the basis vector $|n\rangle$ associated with the number $n$ of photons of the OS field. It is sufficient to restrict the calculation within the Floquet block $|n=0\rangle$ for $X+X$, and $|n=-1\rangle$ for $X+b$ [46]. For bosonic molecules, the dressed symmetrized basis functions are related to the unsymmetrized ones by

$$
\begin{aligned}
& \left|e_{1}, j_{1}, p_{1}, e_{2}, j_{2}, p_{2}, j_{12}, \ell, J, M\right\rangle|n\rangle \\
& =\frac{1}{\sqrt{2\left(1+\delta_{e_{1} e_{2}} \delta_{j_{1} j_{2}} \delta_{p_{1} p_{2}}\right)}} \\
& \quad \times\left[\left|e_{1}\left(j_{1}, p_{1}\right), e_{2}\left(j_{2}, p_{2}\right), j_{12}, \ell, J, M\right\rangle|n\rangle\right. \\
& \left.\quad+\varepsilon\left|e_{2}\left(j_{2}, p_{2}\right), e_{1}\left(j_{1}, p_{1}\right), j_{12}, \ell, J, M\right\rangle|n\rangle\right],
\end{aligned}
$$

with $\varepsilon=(-1)^{j_{1}+j_{2}-j_{12}+\ell}$. It imposes $\varepsilon=1$ for molecules in the same rovibrational level of the same electronic state [45].

The Hamiltonian (1) expressed in the basis (2) generates a set of close-coupled Schrödinger equations which are numerically solved [46] with a log-derivative method [56,57] over the interval $\left[R_{\min } ; R_{\max }\right]=[10$ a.u.; 10000 a.u. $]$. We assume that the scattering flux toward short distances is fully absorbed as in Ref. [58], as successfully applied to simulate the observed reactive or nonreactive ultracold molecular collisions [13,14,22]. The $S$ matrix is extracted at $R_{\max }$, and rate coefficients are obtained.

We consider two ${ }^{23} \mathrm{Na}^{87} \mathrm{Rb}$ molecules prepared in their absolute ground state $\mid e_{1}=X, j_{1}=0, p_{1}=1, e_{2}=X$, $\left.j_{2}=0, p_{2}=1, j_{12}=0, \ell, J=\ell, M=0\right\rangle|n=0\rangle$. In typical experimental conditions [18], the temperature $T=$ $E_{\mathrm{col}} / k_{B} \approx 400 \mathrm{nK}$ only involves $\ell=0,2$. In the field-free case, the states correlated to the $\left(j_{X}=0\right)+\left(j_{X}=0\right)$ asymptote are restricted to $J=0$ and $J=2$. The DDI couples different $j_{i}$ and $\ell$ such that $\left|j_{i}^{\prime}-j_{i}\right|=1$, 


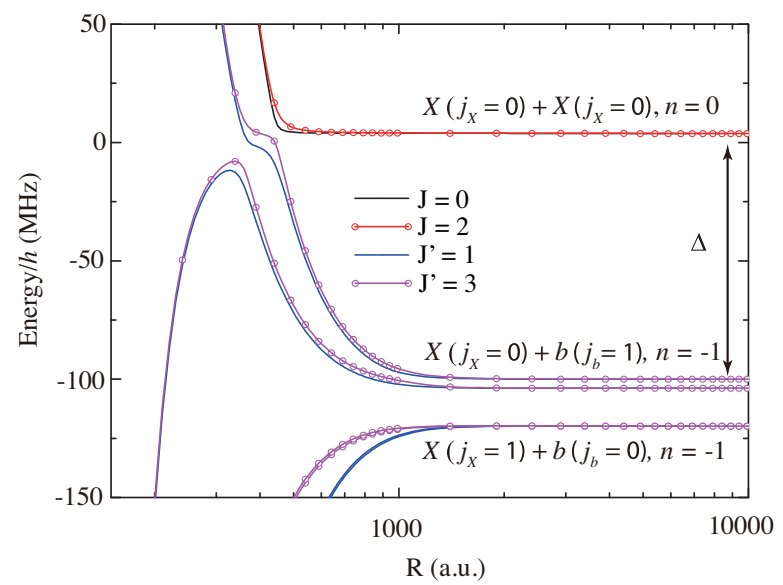

FIG. 2. The dressed adiabatic long-range PECs of ${ }^{23} \mathrm{Na}^{87} \mathrm{Rb}-{ }^{23} \mathrm{Na}^{87} \mathrm{Rb}$ for $\Delta=100 \mathrm{MHz}$ and $\Omega=10 \mathrm{MHz}$, in linear polarization. The Condon point (not displayed here) lies around $R_{C}=400$ a.u.. The curves are labeled with their dominant $J$ character. Note that the asymptotic spacing between the $\left(j_{X}=0\right)+\left(j_{X}=0\right)$ and $\left(j_{X}=0\right)+\left(j_{b}=1\right)$ asymptotes is slightly larger (by $7.7 \mathrm{MHz}$ ) than $\Delta$ due to the presence of the stationary laser field [31]. For the same reason, the $\left(j_{X}=0\right)+$ $\left(j_{b}=1\right)$ asymptote is split by $3.85 \mathrm{MHz}$ as all the relevant states do not interact in the same way due to the laser.

$p_{i} p_{i}^{\prime}=-1$, and $\left|\ell^{\prime}-\ell\right|=0,2$. The long-range adiabatic PECs resulting from the diagonalization of the field-free Hamiltonian [Eq. (1) without $\hat{H}_{f}$ and $\hat{H}_{I}$ ] in the basis of Eq. (2) (without $|n=0\rangle$ ) are presented in the Supplemental Material [46]. They are calculated for $j_{i} \in\left[0, j_{\max }\right]$ and $\ell \in\left[0, \ell_{\max }\right]$, where $j_{\max }=4$ and $\ell_{\max }=4$ to ensure the convergence of the rate coefficients.

In Fig. 2 we present the ${ }^{23} \mathrm{Na}^{87} \mathrm{Rb}^{23} \mathrm{Na}^{87} \mathrm{Rb}$ dressed adiabatic long-range PECs in the presence of $\pi$ light with $\Delta=100 \mathrm{MHz}$ and $\Omega=10 \mathrm{MHz}$. The PECs are similar for $\sigma^{ \pm}$light [46]. The states correlated to $\left(j_{X}=0\right)+\left(j_{X}=0\right)$ with $n=0$ (with quantum numbers $J$ and $M$ ) are directly coupled to those correlated to $\left(j_{X}=0\right)+\left(j_{b}=1\right)$ with $n=-1$ (with quantum numbers $J^{\prime}$ and $M^{\prime}$ ). Note that the $\left(j_{X}=1\right)+\left(j_{b}=0\right)$ asymptote is distant by about $h \times$ $20 \mathrm{MHz}$ from $\left(j_{X}=0\right)+\left(j_{b}=1\right)$ due to the almost identical values of the $b$ and $X$ rotational constants [46], so that it must be included in the calculations. The DDI induces the strong repulsive character of the upper manifold. Restricting the dressed-state approach to singlephoton transitions, the laser-induced couplings obey the electric dipole selection rules, $\left|J^{\prime}-J\right|=1$ and $M^{\prime}=M$ (resp. $\left|J^{\prime}-J\right|=0,1$ and $M^{\prime}=M \pm 1$ ) for $\pi$ (resp. $\sigma^{ \pm}$) light [39]. We performed our study for $M=0$, as the entrance channel is $\left(j_{X}=0\right)+\left(j_{X}=0\right)$ dominated by the $s$ wave $(\ell=0)$ at ultracold energies.

The shielding possibility is clearly visible in Fig. 2. The dressed entrance channels correlated to $\left(j_{X}=0\right)+$ $\left(j_{X}=0\right)$ now undergo avoided crossings with the repulsive channels correlated to $\left(j_{X}=0\right)+\left(j_{b}=1\right)$ so that the incoming flux is repelled, as demonstrated below. The picture is similar for both $\sigma^{ \pm}$and $\pi$ light, in striking contrast with the molecular MW shielding $[27,28]$ or with the atomic case [33]. Indeed, the permutation symmetry selection rules allow the $d$ wave to be coupled to $\left(j_{X}=0\right)+\left(j_{b}=1\right)$ states not only in circular polarization but also in a linear one.

The OS efficiency requires that the elastic collision rate $k_{\mathrm{el}}$ induced by the repulsive PECs in the entrance channel dominate over the loss rates, namely, $k_{\mathrm{re}}$ for the short-range reactive collisions and $k_{\text {in }}$ for the photoinduced inelastic collisions $\quad\left(j_{X}=0\right)+\left(j_{X}=0\right) \rightarrow\left(j_{X}=0\right)+\left(j_{b}=1\right)$. We look for conditions maximizing the ratio $\gamma=k_{\mathrm{el}} /\left(k_{\mathrm{re}}+k_{\mathrm{in}}\right)$, often referred to as the "good"-todestructive collisional rate ratio [60].

In Fig. 3 we display the variation of the rate coefficients and $\gamma$ with $\Omega$ at fixed $\Delta=100 \mathrm{MHz}$ for both polarizations. In the field-free case, the computed $k_{\mathrm{re}}=4.0 \times$ $10^{-10} \mathrm{~cm}^{3} \mathrm{~s}^{-1}$ is consistent with the experimental total loss rate $\left[4.5(2) \times 10^{-10} \mathrm{~cm}^{3} \mathrm{~s}^{-1}\right]$ and the theoretical one $\left(3.8 \times 10^{-10} \mathrm{~cm}^{3} \mathrm{~s}^{-1}\right)$ reported in Ref. [22]. The computed value $\gamma \sim 0.3$ confirms the inefficiency of evaporative cooling, which requires $\gamma \gtrsim 1000$ [22]. The $k_{\text {re }}$ rate drastically decreases with increasing $\Omega$, stabilizing to $k_{\mathrm{re}} \approx$ $3.0 \times 10^{-17} \mathrm{~cm}^{3} \mathrm{~s}^{-1}$ for $\Omega>40 \mathrm{MHz}$. After reaching a maximal value of about $10^{-10} \mathrm{~cm}^{3} \mathrm{~s}^{-1}$, the $k_{\text {in }}$ value also strongly decreases, down to $5 \times 10^{-14} \mathrm{~cm}^{3} \mathrm{~s}^{-1}$ (resp. $3 \times 10^{-13} \mathrm{~cm}^{3} \mathrm{~s}^{-1}$ ) for circular (resp. linear) polarization at $\Omega=50 \mathrm{MHz}$. The $\gamma$ ratio is as high as 8000 and 2000 for circular and linear polarizations, respectively. We computed a transition dipole moment between $\left(j_{X}=0\right)$ and $\left(j_{b}=1\right)$ equal to 0.1918 a.u. [46] so that this regime is reached for a
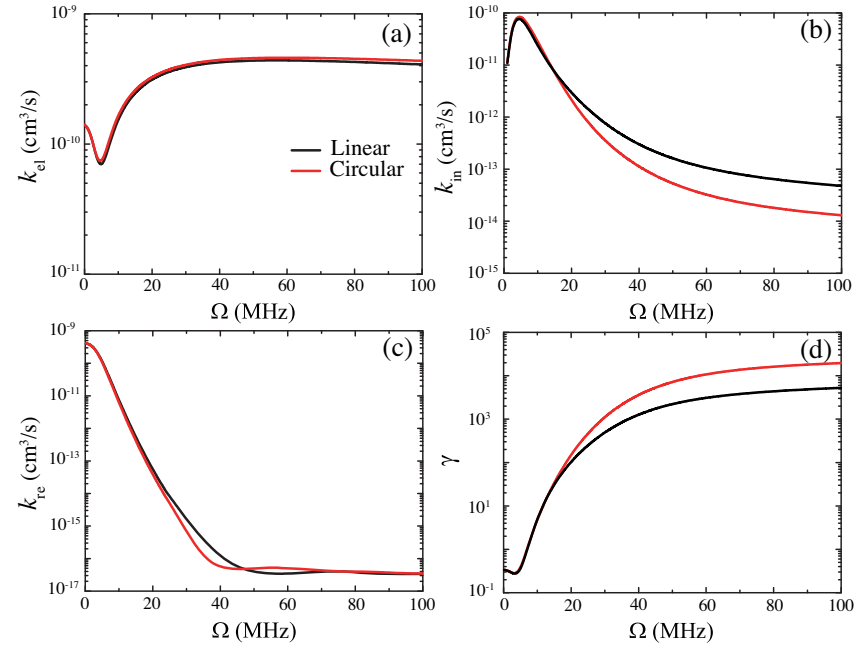

FIG. 3. Variation with the Rabi frequency $\Omega$ of the rate coefficients $k_{\mathrm{el}}, k_{\mathrm{in}}, k_{\mathrm{re}}$ for (a) elastic, (b) photoinduced inelastic, and (c) reactive collisions, respectively, and (d) of the shielding efficiency $\gamma$, for both linear and circular polarizations. The collision energy is $k_{B} \times 400 \mathrm{nK}$ and the laser detuning $\Delta=100 \mathrm{MHz}$. 

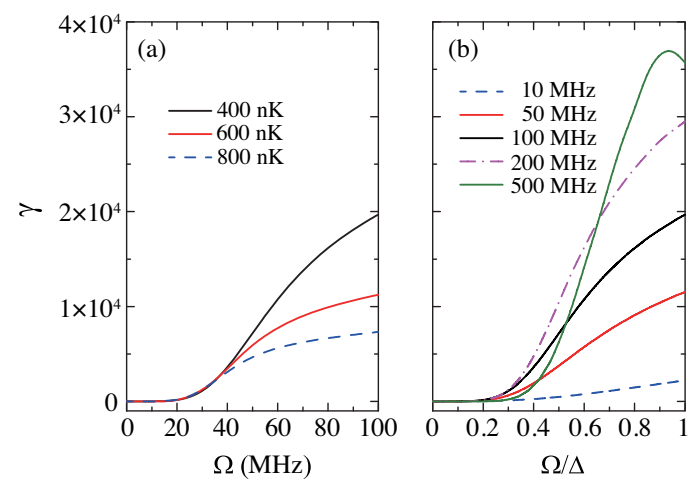

FIG. 4. Variation of $\gamma$ in circularly polarized light (a) with $\Omega$, at $\Delta=100 \mathrm{MHz}$, and for $T=400,600,800 \mathrm{nK}$, and (b) with the ratio $\Omega / \Delta$ (kept $<1$ ), at $T=400 \mathrm{nK}$ for $\Delta=10,50,100,200$, $500 \mathrm{MHz}$.

moderate intensity of about $12 \mathrm{~W} \mathrm{~cm}^{-2}$. Large $\gamma$ values imply dominant elastic collision rate, so that OS should allow an efficient evaporative cooling of the molecules.

The influence of $T$ and $\Delta$ on $\gamma$ is illustrated in Fig. 4 with $\sigma^{ \pm}$light as it appears more efficient (Fig. 3). In Fig. 4(a), $\Delta=100 \mathrm{MHz}$, and we see that $\gamma$ is insensitive to $T$ as long as the reactive rate dominates the inelastic one $(\Omega<20 \mathrm{MHz})$, meaning that the avoided crossing is almost diabatic. When the reactive rate drops down abruptly, the inelastic rate reveals its sensitivity to the details of the avoided crossing: While $k_{\text {in }}$ varies by order of magnitude when $\Omega$ is multiplied by $5, \gamma$ varies at most by a factor of 2 over the same range when changing the temperature by a factor of 2 (reflecting that $E_{\mathrm{col}} \ll h \Omega$ ). In Fig. 4(b), we fixed $T=400 \mathrm{nK}$, and $\gamma$ is displayed for convenience as a function of $\Omega / \Delta$ for various values of $\Delta$, thus, all on the same scale. The restriction $\Omega / \Delta<1$ allows us to keep valid and useful the representation of an avoided crossing (Fig. 2). Again, no significant change is visible for $\Omega / \Delta<0.2$ (the reactive rate dominates), while the details of the avoided crossing are manifested above this value. At $\Delta=500 \mathrm{MHz}, \gamma$ reaches a maximum and then decreases, which is caused by the enhancement of photoinduced inelastic collision from the ground to the excited states. Large $\Delta$ values seem beneficial for shielding, but this implies large $\Omega$ values, which may be problematic in terms of the power of light sources.

As noted in previous works on atoms, the spontaneous emission (SE) from the excited state during the collision is the main potential limitation of OS. If an excited ${ }^{23} \mathrm{Na}^{87} \mathrm{Rb}$ molecule spontaneously emits a photon, it leads to a large increase in kinetic energy, which is likely to damage OS. In this respect, the MW-based shielding is advantageous as SE is negligible for ground-state rotational levels. The present choice of the $b$ state fulfills this criterion: The lifetime of the $j_{b}=1$ level is $\tau_{\gamma}=6.97 \mu$ s [46]. The classical picture of Fig. 1 is useful in this matter. Following Ref. [61], the duration $\tau_{\mathrm{TP}}$ of the classical motion from the Condon
TABLE I. Estimation of $\Omega$ values and corresponding laser intensities $I$ to reach $\gamma=1000$, at $400 \mathrm{nK}$, with $\sigma^{ \pm}$light and $\Delta=100 \mathrm{MHz}$, for bosonic alkali-metal diatomics. The LiNa and $\mathrm{KRb}$ species are not listed as they do not have large enough DDI to allow such a large value of $\gamma$.

\begin{tabular}{lcc}
\hline \hline Species & $\Omega_{\gamma=1000}(\mathrm{MHz})$ & $I_{\gamma=1000}\left(\mathrm{~W} \mathrm{~cm}^{-2}\right)$ \\
\hline${ }^{7} \mathrm{Li}^{39} \mathrm{~K}$ & 225.1 & 3294 \\
${ }^{7} \mathrm{Li}^{87} \mathrm{Rb}$ & 53.8 & 35 \\
${ }^{7} \mathrm{Li}^{133} \mathrm{Cs}$ & 36.1 & 14 \\
${ }^{23} \mathrm{Na}^{39} \mathrm{~K}$ & 92.8 & 265 \\
${ }^{23} \mathrm{Na}^{87} \mathrm{Rb}$ & 29.3 & 6.3 \\
${ }^{23} \mathrm{Na}^{133} \mathrm{Cs}$ & 24.5 & 2.0 \\
${ }^{39} \mathrm{~K}^{133} \mathrm{Cs}$ & 48.8 & 14 \\
${ }^{87} \mathrm{Rb}^{133} \mathrm{Cs}$ & 101.2 & 53 \\
\hline \hline
\end{tabular}

point $R_{C}$ to the turning point $R_{\mathrm{TP}}$ is given by $\int_{R_{C}}^{R_{\mathrm{TP}}}\left\{\left[d R^{\prime}\right] /\left[v\left(R^{\prime}, p\right)\right]\right\}$ with $v\left(R^{\prime}, p\right)$ the classical local velocity which depends on the initial momentum $p_{0}$ and local PEC. The SE probability $P_{\mathrm{SE}}$ during one collision can be estimated as $P_{\mathrm{SE}}=P_{b} e^{-\tau_{\gamma} / \tau_{\mathrm{TP}}}$ where $P_{b}$ is the population in the $b$ excited state. This formula has been successfully employed in atomic trap loss studies and compared with quantum results of Refs. $[62,63]$. Here we found $\tau_{\mathrm{TP}}=$ $0.79 \mathrm{~ns}$ for $\Delta=10 \mathrm{MHz}$ down to $\tau_{\mathrm{TP}}=1.2 \mathrm{ps}$ for $\Delta=500 \mathrm{MHz}$, indeed negligible compared to $\tau_{\gamma}$.

We predict that OS is also valid for all heteronuclear alkali-metal diatoms except $\mathrm{LiNa}$ and $\mathrm{KRb}$ which do not have a large enough PDM, and thus low DDI [43]. Assuming that the DDI is proportional to the product of the PDM of the colliding molecules, one can scale it along the series of species [46] and repeat the same calculations. We found very similar variations of $\gamma$ shifted toward lower or larger values of $\Omega$. In Table I we see that similar shielding efficiency $(\gamma=1000)$ can be obtained for experimentally acceptable laser intensities (except for LiK). Moreover, being very similar in terms of electronic structure, these species are all expected to possess a long-lived electronic $b$ state. Therefore, evaporative cooling appears as experimentally feasible for all the reported species.

Just like in Refs. [27,28], the present proposal is formulated in free space. We demonstrate in the Supplemental Material [46] that a trapping laser and a static magnetic field with a sufficient magnitude do not alter the OS. However, most ongoing experiments with dipolar particles are intended to exemplify anisotropic interactions when they are exposed to a static electric field. Here an electric field as weak as about $200 \mathrm{~V} / \mathrm{cm}$ splits the closely spaced $j_{X}=0+j_{b}=1$ and $j_{X}=1+j_{b}=0$ manifolds and induces the mixing of sublevels within the same $M$ subspace. Our preliminary investigation indicates that the barrier used for OS surely exists for any field intensity due to the interplay of DDI within these two manifolds. We do expect a good OS efficiency even in the presence of an 
electric field, provided that the OS laser frequency is properly tuned. For instance, at $1.5 \mathrm{kV} / \mathrm{cm}$, typical of ongoing experiments, the OS laser frequency could be changed by about $500 \mathrm{MHz}$ compared to the field-free case. Further calculations including an electric field will be implemented in our next work.

We acknowledge support from the BLUESHIELD ANRproject (Grant No. ANR-14-CE34-0006 from "Agence Nationale de la Recherche"), by "Investissements d'Avenir" LabEx PALM (ANR-10LABX-0039-PALM) [64], by Region Ile-de-France in the framework of DIM SIRTEQ [65], and by a Programme Hubert Curien "BALATON" (Campus France Grant No. 41919RK and TéT-Fr Grant No. 2018.2.1.13). We thank O. Atabek, M. Desouter, E. Luc, G. Quéméner, M. Raoult, and D. Wang for stimulating discussions. Calculations have been performed at the computing center MésoLUM (LUMAT FR2764) and HPC resources from DNUM CCUB (Centre de Calcul de l'Université de Bourgogne).

[1] M. A. Baranov, Phys. Rep. 464, 71 (2008).

[2] I. Bloch, J. Dalibard, and W. Zwerger, Rev. Mod. Phys. 80, 885 (2008).

[3] T. Lahaye, C. Menotti, L. Santos, M. Lewenstein, and T. Pfau, Rep. Prog. Phys. 72, 126401 (2009).

[4] I. Bloch, J. Dalibard, and S. Nascimbène, Nat. Phys. 8, 267 (2012).

[5] P. Schwerdfeger Ultracold Gases and Quantum Information: Lecture Notes of the Les Houches Summer School in Singapore (Oxford University Press, Amsterdam, 2009).

[6] J. Doyle, B. Friedrich, R. Krems, and F. Masnou-Seeuws, Eur. Phys. J. D 31, 149 (2004).

[7] L. D. Carr and J. Ye, New J. Phys. 11, 055009 (2009).

[8] O. Dulieu and C. Gabbanini, Rep. Prog. Phys. 72, 086401 (2009).

[9] G. Quéméner and P. S. Julienne, Chem. Rev. 112, 4949 (2012).

[10] S. A. Moses, J. P. Covey, M. T. Miecnikowski, D. S. Jin, and J. Ye, Nat. Phys. 13, 13 (2017).

[11] J. L. Bohn, A. M. Rey, and J. Ye, Science 357, 1002 (2017).

[12] K. Matsuda, L. De Marco, J.-R. Li, W. G. Tobias, G. Valtolina, G. Quéméner, and J. Ye, arXiv:2009.07461.

[13] S. Ospelkaus, K.-K. Ni, D. Wang, M. H. G. de Miranda, B. Neyenhuis, G. Quéméner, P. S. Julienne, J. Bohn, D. S. Jin, and J. Ye, Science 327, 853 (2010).

[14] K.-K. Ni, S. Ospelkaus, D. Wang, G. Quéméner, B. Neyenhuis, M. H. G. de Miranda, J. L. Bohn, J. Ye, and D. S. Jin, Nature (London) 464, 1324 (2010).

[15] T. Takekoshi, L. Reichsöllner, A. Schindewolf, J. M. Hutson, C. R. LeSueur, O. Dulieu, F. Ferlaino, R. Grimm, and H.-C. Nägerl, Phys. Rev. Lett. 113, 205301 (2014).

[16] P. K. Molony, P. D. Gregory, Z. Ji, B. Lu, M. P. Köppinger, C. R. Le Sueur, C. L. Blackley, J. M. Hutson, and S. L. Cornish, Phys. Rev. Lett. 113, 255301 (2014).
[17] P. D. Gregory, M. D. Frye, J. A. Blackmore, E. M. Bridge, R. Sawant, J. M. Hutson, and S. L. Cornish, Nat. Commun. 10, 3104 (2019).

[18] M. Guo, B. Zhu, B. Lu, X. Ye, F. Wang, R. Vexiau, N. Bouloufa-Maafa, G. Quéméner, O. Dulieu, and D. Wang, Phys. Rev. Lett. 116, 205303 (2016).

[19] J. W. Park, S. A. Will, and M. W. Zwierlein, Phys. Rev. Lett. 114, 205302 (2015).

[20] F. Seeßelberg, N. Buchheim, Z.-K. Lu, T. Schneider, X.-Y. Luo, E. Tiemann, I. Bloch, and C. Gohle, Phys. Rev. A 97, 013405 (2018).

[21] P. S. Żuchowski and J. M. Hutson, Phys. Rev. A 81, 060703 (R) (2010).

[22] X. Ye, M. Guo, M. L. González-Martínez, G. Quéméner, and D. Wang, Sci. Adv. 4, eaaq0083(2018).

[23] M.-G. Hu, Y. Liu, D. D. Grimes, Y.-W. Lin, A. H. Gheorghe, R. Vexiau, N. Bouloufa-Maafa, O. Dulieu, T. Rosenband, and K.-K. Ni, Science 366, 1111 (2019).

[24] Y. Liu, M.-G. Hu, M. A. Nichols, D. D. Grimes, T. Karman, H. Guo, and K.-K. Ni, Nat. Phys. https://doi.org/10.1038/ s41567-020-0968-8 (2020).

[25] A. Christianen, M. W. Zwierlein, G. C. Groenenboom, and T. Karman, Phys. Rev. Lett. 123, 123402 (2019).

[26] P. D. Gregory, J. A. Blackmore, S. L. Bromley, and S. L. Cornish, Phys. Rev. Lett. 124, 163402 (2020).

[27] T. Karman and J. M. Hutson, Phys. Rev. Lett. 121, 163401 (2018).

[28] L. Lassablière and G. Quéméner, Phys. Rev. Lett. 121, 163402 (2018).

[29] A. V. Avdeenkov, New J. Phys. 17, 045025 (2015).

[30] T. Karman, Phys. Rev. A 101, 042702 (2020).

[31] R. Napolitano, J. Weiner, and P. S. Julienne, Phys. Rev. A 55, 1191 (1997).

[32] S. Bali, D. Hoffmann, and T. Walker, Europhys. Lett. 27, 273 (1994).

[33] L. Marcassa, S. Muniz, E. de Queiroz, S. Zilio, V. Bagnato, J. Weiner, P. S. Julienne, and K. A. Suominen, Phys. Rev. Lett. 73, 1911 (1994).

[34] K.-A. Suominen, K. Burnett, P. S. Julienne, M. Walhout, U. Sterr, C. Orzel, M. Hoogerland, and S. L. Rolston, Phys. Rev. A 53, 1678 (1996).

[35] D. Hoffmann, S. Bali, and T. Walker, Phys. Rev. A 54, R1030 (1996).

[36] S. C. Zilio, L. Marcassa, S. Muniz, R. Horowicz, V. Bagnato, R. Napolitano, J. Weiner, and P. S. Julienne, Phys. Rev. Lett. 76, 2033 (1996).

[37] S. R. Muniz, L. G. Marcassa, R. Napolitano, G. D. Telles, J. Weiner, S. C. Zilio, and V. S. Bagnato, Phys. Rev. A 55, 4407 (1997).

[38] J. Weiner, V. S. Bagnato, S. C. Zilio, and P. S. Julienne, Rev. Mod. Phys. 71, 1 (1999).

[39] K.-A. Suominen, M. J. Holland, K. Burnett, and P. Julienne, Phys. Rev. A 51, 1446 (1995).

[40] K. A. Suominen, K. Burnett, and P. S. Julienne, Phys. Rev. A 53, R1220 (1996).

[41] O. Docenko, M. Tamanis, R. Ferber, E. A. Pazyuk, A. Zaitsevskii, A. V. Stolyarov, A. Pashov, H. Knöckel, and E. Tiemann, Phys. Rev. A 75, 042503 (2007).

[42] M. Lepers, R. Vexiau, M. Aymar, N. Bouloufa-Maafa, and O. Dulieu, Phys. Rev. A 88, 032709 (2013). 
[43] R. Vexiau, M. Lepers, M. Aymar, N. Bouloufa-Maafa, and O. Dulieu, J. Chem. Phys. 142, 214303 (2015).

[44] M. Lepers and O. Dulieu, in Cold Chemistry: Molecular Scattering and Reactivity Near Absolute Zero edited by O. Dulieu and A. Osterwalder(The Royal Society of Chemistry, London, 2018), pp. 150-202.

[45] H. Li, G. Quéméner, J. F. Wyart, O. Dulieu, and M. Lepers, Phys. Rev. A 100, 042711 (2019).

[46] See Supplemental Material at http://link.aps.org/ supplemental/10.1103/PhysRevLett.125.153202 for the description of long-range potential curves of $\mathrm{NaRb}-\mathrm{NaRb}$ molecules, the dressed potential -energy curves between two $\mathrm{NaRb}$ molecules in circular polarization, the hyperfine structure in b state, the optical shielding for trapped ultracold molecules, the short-range boundary condition in the log-derivative method, and the optical shielding for other alkali-metal dipolar species, which includes Refs. [46-54].

[47] M. Aymar and O. Dulieu, J. Chem. Phys. 122, 204302 (2005).

[48] J. Aldegunde and J. M. Hutson, Phys. Rev. A 96, 042506 (2017).

[49] A. Orbán, R. Vexiau, O. Krieglsteiner, H.-C. Nägerl, O. Dulieu, A. Crubellier, and N. Bouloufa-Maafa, Phys. Rev. A 92, 032510 (2015).

[50] A. Orbán, T. Xie, R. Vexiau, O. Dulieu, and N. BouloufaMaafa, J. Phys. B 52, 135101 (2019).
[51] M. Mayle, B. P. Ruzic, and J. L. Bohn, Phys. Rev. A 85, 062712 (2012).

[52] R. Vexiau, D. Borsalino, M. Lepers, A. Orbán, M. Aymar, O. Dulieu, and N. Bouloufa-Maafa, Int. Rev. Phys. Chem. 36, 709 (2017).

[53] M. Aymar and O. Dulieu, Mol. Phys. 105, 1733 (2007).

[54] M. Aymar, O. Dulieu, and F. Spiegelman, J. Phys. B 39, S905 (2006).

[55] C. Cohen-Tannoudji, J. Dupont-Roc, and G. Grynberg, in Atom-Photon Interactions: Basic Processes and Applications (Wiley-VCH, New York, 1998).

[56] B. Johnson, J. Comput. Phys. 13, 445 (1973).

[57] I. Tuvi and Y. B. Band, J. Chem. Phys. 99, 9697 (1993).

[58] G. Wang and G. Quéméner, New J. Phys. 17, 035015 (2015).

[59] X. Ye, M. Guo, M. L. González-Martínez, G. Quéméner, and D. Wang, Sci. Adv. 4 (2018).

[60] M. L. González-Martínez, J. L. Bohn, and G. Quéméner, Phys. Rev. A 96, 032718 (2017).

[61] K.-A. Suominen, J. Phys. B 29, 5981 (1996).

[62] P. S. Julienne, K.-A. Suominen, and Y. Band, Phys. Rev. A 49, 3890 (1994).

[63] H. M. J. M. Boesten and B. J. Verhaar, Phys. Rev. A 49, 4240 (1994).

[64] www.sirteq.org.

[65] www.sirteq.org. 